\title{
Sparse solutions of optimal control via Newton method for under-determined systems
}

\author{
Boris Polyak • Andrey Tremba
}

Received: date / Accepted: date

\begin{abstract}
We focus on finding sparse and least- $\ell_{1}$-norm solutions for unconstrained nonlinear optimal control problems. Such optimization problems are non-convex and non-smooth, nevertheless recent versions of Newton method for under-determined equations can be applied successively for such problems.
\end{abstract}

Keywords Optimal control, Sparse control, Newton method, Underdetermined equations, $\ell_{1}$-norm

\section{Introduction}

The simplest optimal control problem has the form

$$
\begin{gathered}
\min \int_{0}^{T} F(x, u, t) d t \\
\dot{x}=\phi(x, u, t), x(0)=a, x(T)=b \in \mathbb{R}^{m}, u(t) \in \mathbb{R}^{q}
\end{gathered}
$$

where there are no constraints on state $x(t)$ and control $u(t)$. There are numerous classical methods for solving the problem such as gradient method, dynamic programming, maximum principle etc. see $[2,3,8,12,13,14,20$. However these methods are oriented on smooth functionals. Our problems of interest are sparse controls, i.e. solutions with small number of non-zero components. Such solutions naturally arise in $L_{1}$ control, where the performance

This work was supported by Russian Science Foundation (project 16-11-10015).

B. Polyak

Institute for Control Sciences, Profsoyuznaya 65, 117997 Moscow, Russia, E-mail: boris@ipu.ru

A. Tremba

Institute for Control Sciences, Profsoyuznaya 65, 117997 Moscow, Russia, E-mail: atremba@ipu.ru 
criteria are $L_{1}$ norms of control (or where $L_{1}$ regularizer is exploited). The $L_{1}$ norm is $\int_{t=0}^{T}\|u(t)\|_{1} d t$, it involves vector $\ell_{1}$-norm (Manhattan, 1-norm) $\|u(t)\|_{1}=\sum_{i=1}^{q}\left|u_{i}(t)\right|$. Examples are minimal fuel control for satellite trajectory optimization, where $L_{1}$ term corresponds to the fuel consumption [1,26], Section 5.5 or so called "maximal hands-off" control [17. If we take finite-dimensional approximation of such problems we arrive to mathematical programming problems with nonlinear (and hence non-convex) equality-type constraints and non-smooth objective function. The $L_{1}$ norm transforms to $\ell_{1}$-norm of the extended vector of (discretized) control. There are no standard algorithms for such hard problems. Of course situation is much simpler if the state equations are linear, then linear programming technique can be applied, see 25] and references therein.

The contributions of the paper are twofold. First, we exhibit that recently developed by the authors Newton-like method for under-determined equations 23. 24 can be highly effective for solving nonlinear optimal control problems. That is, we consider right-hand side condition $x(T)=b$ as $m$ nonlinear equations with variables $u$ (the number of variables $n$ depends on discretization and in general is much larger $m$ ). Above mentioned method iteratively solves linearized equation, finding solution with minimal norm. Second, we apply the method to find sparse solutions. This is due the flexibility of the proposed method to various norms. By choosing $L_{1}$ norm we can find sparse controls. Recent paper [19] also exploits methods from [23,24] for optimal control, but with $L_{2}$ norm of approximations, thus the solutions are not sparse.

Theoretically, the solution of the problem (1) with $L_{1}$ terms may contain impulses, e.g. a combination of delta-functions. However, differential equation is discretized in practice, and the following problem with embedded difference equation is considered

$$
\begin{gathered}
\min F(\{x\},\{u\}) \\
x[j+1]=f_{j}(x[j], u[j]), j=0, \ldots, N-1, \\
x[0]=a, x[N]=b \in \mathbb{R}^{m},
\end{gathered}
$$

with functions $f_{j}: \mathbb{R}^{m+q} \rightarrow \mathbb{R}^{m}$. The functions $f_{j}$ are assumed to be differentiable on both arguments, while $F$ can be non-differentiable. Notice that the dimension of the variables in the problem becomes very large: $q N$, with $N \approx T / h \gg 1, h$ being (small) discretization step-size.

If the objective equals $L_{1}$ norm of control in continuous-time setup, it becomes simple $\ell_{1}$ norm in the discretized problem statement:

$$
F(\{x\},\{u\})=\sum_{j=0}^{N-1}\|u[j]\|_{1} .
$$

The function summarizes absolute values of all components for all time instances. In algorithmic approach, we deal with the discretized equations only. 
Another objective function is the total number of non-zero components, counted through all time instants:

$$
F(\{x\},\{u\})=\text { total \# of non-zero components in }\{u[j]\}_{j=0, \ldots, N-1} \text {. }
$$

We aim to solve problem (2)-(3) with one of the above objective functions. Running ahead, the optimization cannot be done exactly, but an approximate algorithm is proposed, based on the specially fitted version of Newton method. We discuss its applicability and properties. It appears that this approach can manage sparsity constraint (5) directly.

The structure of the paper is as follows. In Section 2 we describe classical Newton method and its versions for under-determined equations. Section 3 contains main results of the paper and addresses applications of the methods to sparse control. There are several versions of the algorithms to get sparse solutions. Results of numerical simulation are provided in Section 4.

\section{Constraint Equation and Newton method}

In this section equation (3) and its solution are considered. It is convenient to vectorize the whole control sequence $\{u[j]\}, j=0, \ldots, N-1$, stacking individual vectors $u[j] \in \mathbb{R}^{q}$ into a big one. Then the control sequence $\{u[k]\}$ is represented by the single vector variable $u=\left(u[0]^{T}, u[1]^{T}, \ldots, u[N-1]^{T}\right)^{T} \in \mathbb{R}^{q N}$. Let's denote its dimension as $n=q N$.

Equations (3) can be presented in the form of the single equation

$$
P(u) \doteq x[N](u)-b=0 \in \mathbb{R}^{m}, u \in \mathbb{R}^{n} .
$$

The function $P(u)$ is easily calculated by applying the recursive relation of (3):

$$
x[j+1]=f_{j}(x[j], u[j]), j=0, \ldots, N-1, x[0]=a .
$$

Equation (6) is strongly under-determined, as $m \ll n=q N$. If the functions $f_{j}$ are differentiable, so is the function $P$. Its derivative is easily obtained by the chain rule applied to $P(u)=f_{N-1}\left(f_{N-2}(\ldots, u[N-2]), u[N-1]\right)$, see an example below in Section 3 .

\subsection{Regular Newton method and its versions}

One of the most popular generic algorithms for solving smooth nonlinear equations is Newton method, which uses the idea of the linearization of $P(u)$ at each iteration [7,11,18]:

$$
u^{k+1}=u^{k}-\gamma_{k}\left(P^{\prime}\left(u^{k}\right)\right)^{-1} P\left(u^{k}\right), k=0,1, \ldots
$$

The formulae includes both pure $\left(\gamma_{k} \equiv 1\right)$ and damped $\left(\gamma_{k} \leq 1\right)$ variants of Newton method. 
It is known that if Newton method converges, then its convergence rate is quadratic $\left\|P\left(u^{k+1}\right)\right\| \leq c_{1}\left\|P\left(u^{k}\right)\right\|^{2}$. Famous Newton-Kantorovich theorem impose semi-local conditions, ensuring that pure Newton method converges [10.

The theorem operates with four entities, matching four assumptions:

A. Let $\mu_{0}$ be a constant, describing non-degeneracy $P^{\prime}$ at a single point $u^{0}$.

B. Let $L$ be a constant, describing variability of $P^{\prime}$ around $u^{0}$.

C. Let $\rho$ be the radius of the abovementioned "around $u^{0}$ " area, where the constant $L$ is valid.

D. And let $s$ be the size of initial residual, e.g. $\left\|P\left(u^{0}\right)\right\|$.

We intentionally avoid unneccessary formalization of the constants here, to clarify them with respect to under-determined case later. In a particular case, $\mu_{0}$ is the least singular value of $P^{\prime}\left(u^{0}\right)$ and $L$ is the Lipschitz constant of the derivative. It is sufficient for $P$ to be differentiable around $u^{0}$, but for simplicity of statements we assume that $P$ is differentiable everywhere.

We also omit the exact formulation of the Newton-Kantorovich theorem, but its virtue is the following:

If $\mathbf{A}, \mathbf{B}, \mathbf{C}, \mathbf{D}$ hold, and the following conditions

$$
h \doteq \frac{L}{\mu_{0}^{2}} s<\frac{1}{2}, \quad \text { and } \frac{1-\sqrt{1-2 h}}{h} \frac{s}{\mu_{0}} \leq \rho,
$$

hold true, then pure Newton method converges with quadratic rate [10]. There are multiple results on rigorous convergence conditions, cf. surveys [22,28].

Another theorem, by Mysovskikh [10, exploits the following assumption $\mathbf{A}^{\prime}$ instead of $\mathbf{A}$ :

$\mathbf{A}^{\prime}$. Let $\mu$ be a constant, uniformly describing non-degeneracy of $P^{\prime}$ around point $u^{0}$.

The typical result [21, Corollary 1] reads as: If $\mathbf{A}^{\prime}, \mathbf{B}, \mathbf{C}, \mathbf{D}$ hold, and inequalities

$$
h \doteq \frac{L}{\mu^{2}} s<2, \quad \text { and } \frac{2 \mu}{L} H_{0}\left(\frac{h}{2}\right)<\rho,
$$

are satisfied, then pure Newton method converges to the solution $u^{*}$ with quadratic convergence rate. The monotonically increasing function $H_{0}(\delta)=$ $\sum_{\ell=0}^{\infty} \delta^{\left(2^{\ell}\right)}$ is an infinite sum of double exponents.

In both cases, from conditions (7), (8) it follows, that convergence radius $s=\left\|P\left(u^{0}\right)\right\|$ is limited for fixed constants $L$ and $\mu$ (or $\left.\mu_{0}\right)$. It means that the convergence of pure Newton method is always local.

Damped Newton method is a very natural choice for situations when pure Newton method fails. The damping parameter $\gamma_{k} \leq 1$ allows to make non-unit step-size in Newton direction $-w^{k}=-\left(P^{\prime}\left(u^{k}\right)\right)^{-1} P\left(u^{k}\right)$, and due to the stepsize tuning damped Newton method may be converging globally. Algorithms for adjusting $\gamma_{k}$ can be found in [5, 18]. 
2.2 Newton method for under-determined equations

Regular Newton method assumes the existence of the inverse operator $\left(P^{\prime}(u)\right)^{-1}$ near the solution and initial point, so direct application of the method is restricted to the case $n=m$.

The first version of the Newton method for arbitrary $m \neq n$ case has been proposed in 4], see also [15]:

$$
u^{k+1}=u^{k}-\left(P^{\prime}\left(u^{k}\right)\right)^{\dagger} P\left(u^{k}\right),
$$

here $A^{\dagger}$ is the Moore-Penrose pseudo-inverse of a matrix $A$. This includes under-determined case $m<n$. Other versions of the method for under-determined equations can be found in $9,16,27$ and references therein. We rely on the approach, proposed in our previous works [23,24]. Its general form looks as follows

$$
\begin{aligned}
& w^{k}=\arg \min _{P^{\prime}\left(u^{k}\right) w=P\left(u^{k}\right)}\|w\|, \\
& u^{k+1}=u^{k}-\gamma_{k} w^{k} .
\end{aligned}
$$

This description unifies under-determined and regular case. Two key properties of 10 are:

1. freedom of choosing a norm of $w$ to be minimized, and

2. freedom of step-size $\gamma_{k}$ choice.

The first property is completely absent in regular, well-defined systems of equations with $n=m$. For under-determined case, if norm is Euclidean, $w^{k}=$ $\left(P^{\prime}\left(u^{k}\right)\right)^{\dagger} P\left(u^{k}\right)$, and we arrive to the method with pseudo-inverse operator (9). For our purposes (finding sparse solutions) we use method in form 10 with $\ell_{1}$-norm, with zero initial condition $u^{0}=0$. Then the auxiliary problem of finding $w^{k}$ direction is equivalent to linear programming one.

In [23, 24] the authors proposed two novel, alternative strategies for choosing damping parameter $\gamma_{k}$ :

$$
\gamma_{k}=\min \left\{1, \frac{\mu^{2}}{L\left\|P\left(x^{k}\right)\right\|}\right\}
$$

and

$$
\gamma_{k}=\min \left\{1, \frac{\left\|P\left(u^{k}\right)\right\|}{L\left\|w^{k}\right\|^{2}}\right\}
$$

Whenever needed, these strategies perform damped Newton steps at the beginning, switching to pure Newton method later. There are adaptive variants of the strategies, requiring no knowledge of the parameters $\mu, L$ (of $\mathbf{A}^{\prime}, \mathbf{B}$ ), see Algorithm in Section 3 . 
2.3 Convergence Results

For an under-determined equation $P(u)=0, P: \mathbb{R}^{n} \rightarrow \mathbb{R}^{m}$ with $m \leq n$, we are to establish few results for "sparse-Newton" method 10 in the form

$$
\begin{aligned}
& w^{k}=\arg \min _{P^{\prime}\left(u^{k}\right) w=P\left(u^{k}\right)}\|w\|_{1}, \\
& u^{k+1}=u^{k}-\gamma_{k} w^{k} .
\end{aligned}
$$

with the initial condition $u^{0}$. Main topics of interest is the behavior of the objective functions (4) and (5).

Assumptions/constants for the specific case are the following:

C. $\rho$ is the radius of the $\ell_{1}$-ball around the initial point: $B_{\rho}=\left\{u:\left\|u-u^{0}\right\|_{1} \leq\right.$ $\rho$.

A. $\mu_{0}>0$ is a constant, satisfying $\left\|\left(P^{\prime}\left(u^{0}\right)\right)^{T} h\right\|_{1} \geq \mu_{0}\|h\|_{\infty}$ for all $h \in \mathbb{R}^{m}$.

$\mathbf{A}^{\prime} \cdot \mu>0$ is a constant, satisfying $\left\|\left(P^{\prime}(u)\right)^{T} h\right\|_{1} \geq \mu\|h\|_{\infty}$ for all $h \in \mathbb{R}^{m}$ and all $u \in B_{\rho}$.

B. Let $L$ be the Lipschitz constant of $P^{\prime}(u)$ :

$$
\left\|P^{\prime}\left(u^{a}\right)-P^{\prime}\left(u^{b}\right)\right\|_{\infty} \leq L\left\|u^{a}-u^{b}\right\|_{1}, \quad \forall u^{a}, u^{b} \in B_{\rho} .
$$

D. $s$ is the $\ell_{\infty}$-norm of the initial residual, i.e. $s=\left\|P\left(u^{0}\right)\right\|_{\infty}$.

First we consider pure "sparse-Newton" method with $\gamma_{k} \equiv 1$.

If $\mathbf{A}, \mathbf{B}, \mathbf{C}, \mathbf{D}$ hold alongside with (7), then the "sparse-Newton" method (13) converges to a solution $u^{*}$, and $\left\|u^{0}-u^{*}\right\|_{1} \leq \frac{\mu_{0}}{L}\left(1-\sqrt{1-\frac{2 L}{\mu_{0}^{2}} s}\right)$.

If $\mathbf{A}^{\prime}, \mathbf{B}, \mathbf{C}, \mathbf{D}$ hold alongside with (8), then the "sparse-Newton" method 13. converges to a solution $u^{*}$, and $\left\|u^{0}-u^{*}\right\|_{1} \leq \frac{\mu}{L} H_{0}\left(\frac{L}{2 \mu^{2}} s\right)$.

The results follow from (7), (8) by virtue of analysis 10 for specified norms. In both cases the initial residual $s$ is limited by $\frac{\mu_{0}^{2}}{2 L}$ or $\frac{2 \mu^{2}}{L}$.

Newton algorithm 10 with adaptive step-size 11 extends the limits for $s$, and being potentially unlimited allows global convergence. Convergence conditions extend (8), cf. 23] for details. Particularly, Newton algorithm with step-size 11 makes not more than

$$
k_{\max }=\max \left\{0,\left\lceil\frac{2 L}{\mu^{2}} s\right\rceil-2\right\}
$$

damped Newton iterations, followed by pure Newton iterations. At $k$-th step the following estimates for the rate of convergence hold:

$$
\left\|u^{k}-u^{*}\right\|_{1} \leq \begin{cases}\frac{\mu}{L}\left(k_{\max }-k+2 H_{0}\left(\frac{\bar{v}}{2}\right)\right), & k<k_{\max } \\ \frac{2 \mu}{L} H_{k-k_{\max }}\left(\frac{\bar{v}}{2}\right), & k \geq k_{\max }\end{cases}
$$

where $\bar{v}=\frac{L}{\mu^{2}} s-\frac{k_{\max }}{2}<1$. 
If started from $u^{0}=0$, the upper estimate for (4) is

$$
\left\|u^{*}\right\|_{1} \leq \frac{\mu}{L}\left(k_{\max }+2 H_{0}\left(\frac{\bar{v}}{2}\right)\right)
$$

Thus if the sparse Newton method converges to the solution, the total number of steps to achieve accuracy $\left\|P\left(u^{k}\right)\right\|_{\infty} \leq \varepsilon$ is estimated as

$$
K_{\varepsilon}=k_{\max }+\left\lceil\log _{2}\left(\log _{\bar{v} / 2}\left(\frac{\varepsilon L}{2 \mu^{2}}\right)\right)\right\rceil .
$$

As the method achieves the given accuracy, sparsity is also present:

Proposition 1 For $u^{0}=0$ there exists an approximate solution $\widehat{u} \doteq u^{K_{\varepsilon}}$ with not more than $K_{\varepsilon} m$ non-zero components, such that $P(\widehat{u})=\|x[T](\widehat{u})-b\|_{\infty} \leq$ $\varepsilon$.

Indeed each of the $w_{i}$ in Newton step 13 is a solution of $\ell_{1}$-optimization problem with $m$ equality constraints. If the constraints are compatible, then there exists a solution $w_{i}$ with $m$ non-zero components only [6, 25], so up to $k$-th step there are not more than $k m$ non-zero components in $u^{k}$.

This proposition connects two terms: Sparsity and $\ell_{1}$-objective function. The latter is a common convex substitute to the "number of non-zero components" criteria, and is commonly used in machine learning, compressed sensing etc. 6. We propose using the same heuristic for objective (5), but iterationwise. Coupled with fast convergence of Newton method, it results in the total sparsity of the solution.

\section{Application to sparse control}

Consider a non-linear discrete-time dynamic system on a finite interval:

$$
x[j+1]=f(x[j])+B u[j], \quad j=0, \ldots, N-1,
$$

with $x[j] \in \mathbb{R}^{m}, u[j] \in \mathbb{R}^{q}$, differentiable function $f: \mathbb{R}^{m} \rightarrow \mathbb{R}^{m}$ and matrix $B$ of proper dimension $q \times m$. Here we use bracketed argument to distinguish time dynamic from algorithmic iterations. For simplicity we restrict ourselves with systems, nonlinear in $x$ and linear in $u$; the extension to general case (3) is obvious.

Given the initial condition $x[0]$ and the terminal point $x[N]=b$, the goal is to find a control sequence $u=\{u[j]\}$ which would satisfy condition $x[N]=b$ via "the least effort control", i.e. solving optimization problem

$$
\|u\|_{1} \rightarrow \min _{P(u)=0}
$$

with $P(u)=x[N](u)-b$. In the case of a non-linear function $f(x)$ the optimization problem (17) is generally very hard to solve. This is due to the equality-type constraint $x[N]=b$ describing essentially non-convex set in the space of control variables. 
The problem (17) has rather interesting property - its solution appears to be sparse, i.e. contains few non-zero elements. However, if a generic optimization algorithm is used for solving (17) straightforwardly, e.g. via (sub)gradient method with projections on the manifold $\{u: P(u)=0\}$, then it meets two obstacles. First, finding the projection is hard problem itself, and second its solution would be non-sparse.

We propose a heuristic algorithm to find an approximate solution of (17). The idea is to put sparsity property of solution in front. New problem is informally described as

Find sparse solution $u: P(u)=0$.

That is we are to find a solution with small number of non-zero components. This can be achieved by multiple ways, for example:

1. Fix all except few coordinates of $u$ to zeros. Then try to solve equation $P(u)=0$ for the free components only. If all combinations are checked, the optimal one may be found as well.

2. Run "sparse-Newton" algorithm (13), which adds few non-zero components into $u$ at each step.

3. Combine two previous approaches: Run "sparse-Newton" algorithm (13) for one or few steps, then fix some components of $u$ based on these steps and solve the system with respect to the selected components.

First approach is hard to implement, because of its combinatorial nature. The second and third approaches rely on special "sparse-Newton" algorithm (13). The idea is to incorporate our desire of sparsity within Newton steps. This can be perfectly done by use of $\ell_{1}$-norm. In the third approach number of non-zero components in $u$ is controlled directly, and it can be applied for treating objective function (5).

Important part of the algorithm is the choice of step-size $\gamma_{k}$. As problem's constants are rarely known, policies (11), 12 are not applicable directly. Let's describe an algorithm, requiring no a-priori knowledge of the constants. It is based on 11.

The algorithm is initialized with scalar parameters $\beta_{0}>0,0<q<1$, stopping condition $\varepsilon>0$, counter $k=0$, zero initial condition $u^{0}=0 \in \mathbb{R}^{n}$, and number $p_{0}=\left\|P\left(u^{0}\right)\right\|_{\infty}$. 


\section{Adaptive sparse-Newton algorithm}

1. Solve LP problem $w^{k}=\arg \min _{P^{\prime}\left(u^{k}\right) w=P\left(u^{k}\right)}\|w\|_{1}$.

2. Evaluate $p_{k+1}=\left\|P\left(u^{k}-\min \left\{1, \frac{\beta_{k}}{p_{k}}\right\} w^{k}\right)\right\|_{\infty}$.

3. If either a) $\beta_{k}<p_{k}$ and $p_{k+1}<p_{k}-\frac{\beta_{k}}{2}$, or b) $\beta_{k} \geq p_{k}$ and $p_{k+1}<\frac{1}{2 \beta_{k}} p_{k}^{2}$ holds, then go to Step 5

4. Update $\beta_{k} \leftarrow q \beta_{k}$ and return to Step 2 without increasing counter.

5. Take $u^{k+1}=u^{k}-\min \left\{1, \frac{\beta_{k}}{p_{k}}\right\} w^{k}$, set $\beta_{k+1}=\beta_{k}$, increase counter $k \leftarrow k+1$.

6. Check stopping condition $p_{k} \leq \varepsilon$. If it holds, return $u^{k}$ as the solution. Otherwise return to Step 1

The algorithm has similar to Proposition 1 sparsity property: After $k$ steps there is not more than $\mathrm{km}$ non-zero components in $u^{k}$. If Newton method converges in few iterations, then resulting $u$ has few non-zero components as well.

The convergence of the adaptive sparse-Newton algorithm is stated in terms of $\beta_{0}, q,\|P(0)\|_{\infty}$ and properties $\mathbf{A}^{\prime}, \mathbf{B}, \mathbf{C}, \mathbf{D}$ of $P$, cf. [23].

\subsection{Calculating derivatives}

Calculating function $P(u)$ and derivative $P^{\prime}(u)$ is an easy task. First, run iterations (16), resulting in intermediate $x[j], j=1, \ldots, N$, which immediately lead to $P(u)=x[N]-b$. Then we apply chain rule to

$$
\begin{aligned}
P(u) & =x[N](u)-b=f(x[N-1])+B u[N-1]-b= \\
& =f(f(x[N-2])+B u[N-2])+B u[N-1]-b=\ldots= \\
& =f(f(f(\ldots)+B u[N-3])+B u[N-2])+B u[N-1]-b .
\end{aligned}
$$

Blocks of partial derivatives $Q_{r}=P_{u[r]}^{\prime}$ contain products of the Jacobian matrices of $f(\cdot)$ as

$Q_{r}=\prod_{j=N-r}^{N-1} f^{\prime}(x[j])=f^{\prime}(x[N-r]) f^{\prime}(x[N-r+1]) \cdot \ldots \cdot f^{\prime}(x[N-1]) B, r=1, \ldots, N-1$,

with $Q_{0}=B$. The derivative $P^{\prime}(u) \in \mathbb{R}^{p \times q N}$ is formed as horizontally concatenated matrix

$$
\begin{aligned}
P^{\prime}(u) & =\left[Q_{N-1}, Q_{N-2}, \ldots, Q_{1}, Q_{0}\right]= \\
& =\left[\prod_{j=1}^{N-1} f^{\prime}(x[j]) B, \ldots, f^{\prime}(x[N-2]) f^{\prime}(x[N-1]) B, f^{\prime}(x[N-1]) B, B\right] .
\end{aligned}
$$


Generic nonlinear discrete system (3) can be treated similarly. The only difference is a more complex routine for the evaluation of $P^{\prime}(u)$.

\section{Example}

Dynamic equation (16) often arises due to discretization of a continuous-time dynamic systems with small step-size. As simple example we consider a mathematical pendulum with friction. In normalized variables of angle $\phi$ and angular speed $\psi=\dot{\phi}$ pendulum's dynamics can be described as

$$
\ddot{\phi}+\alpha \dot{\phi}+\beta \sin (\phi)=u,
$$

or

$$
\left(\begin{array}{c}
\dot{\phi} \\
\dot{\psi}
\end{array}\right)=\left(\begin{array}{c}
\psi \\
-\alpha \psi-\beta \sin (\phi)
\end{array}\right)+\left(\begin{array}{l}
0 \\
1
\end{array}\right) u
$$

The latter equation has discrete counterpart with $\left(x_{1}[j], x_{2}[j]\right) \approx(\phi(j h), \psi(j h))$. It is obtained by Euler forward method with step $h$. Written in the form (16), function $f$ and matrix $B$ are

$$
f(x)=\left(\begin{array}{c}
x_{1}+h x_{2} \\
x_{2}+h\left(-\alpha x_{2}-\beta \sin \left(x_{1}\right)\right)
\end{array}\right), \quad B=\left(\begin{array}{l}
0 \\
1
\end{array}\right) .
$$

For numerical experiments we set $\alpha=0.3, \beta=0.9, h=0.04, N=160, x[0]=$ $(1,0.5)^{T}, b=(0.4,0)^{T}$.

At the first experiment we use sparse-Newton method, following the second approach mentioned above (by running algorithm (13)). It converges in 3 steps, and after the second step $\left\|P\left(x^{k}\right)\right\|_{\infty} \leq 10^{-5}$. At last step machine accuracy is achieved. Trajectories for all control sequences $u^{k}, k=0, \ldots, 3$ are shown as two upper plots on Figure 1. The trajectories of $u^{2}, u^{3}$ are visually non-distinguishable. Components of internal variable $w^{k}$, used in sparseNewton method, are seen on the next three plots. There are only two nonzero components at each iteration indeed. Notice that the magnitudes of the components rapidly decrease over iterations. Resulting control $u^{3}$ is on the bottom plot of Figure 1. It has only 5 non-zero elements with indices $j \in\{99,153,154,158,159\}$. The value of the functional $\left\|u^{3}\right\|_{1}=0.6544$.

For the second experiment we use the third approach with the following tactic: After the first iteration of sparse-Newton method, the nonzero components $w$ are revealed (indices $j \in\{99,159\}$ ). Then we fix all other components of $u$ as zero, and solve well-defined equation $P(u)=0$ as $P(u=$ $\left.\left(0, \ldots, 0, u_{99}, 0, \ldots, 0, u_{159}\right)\right)=P\left(u_{99}, u_{159}\right)=0$. The equation is solved by adaptive Newton method with accuracy $10^{-9}$.

The result of the experiment is on Figure 2. Now the resulting control has functional $\|u\|_{1}=0.6331$, which appears to be less than in the first experiment, with only 2 non-zero components. 

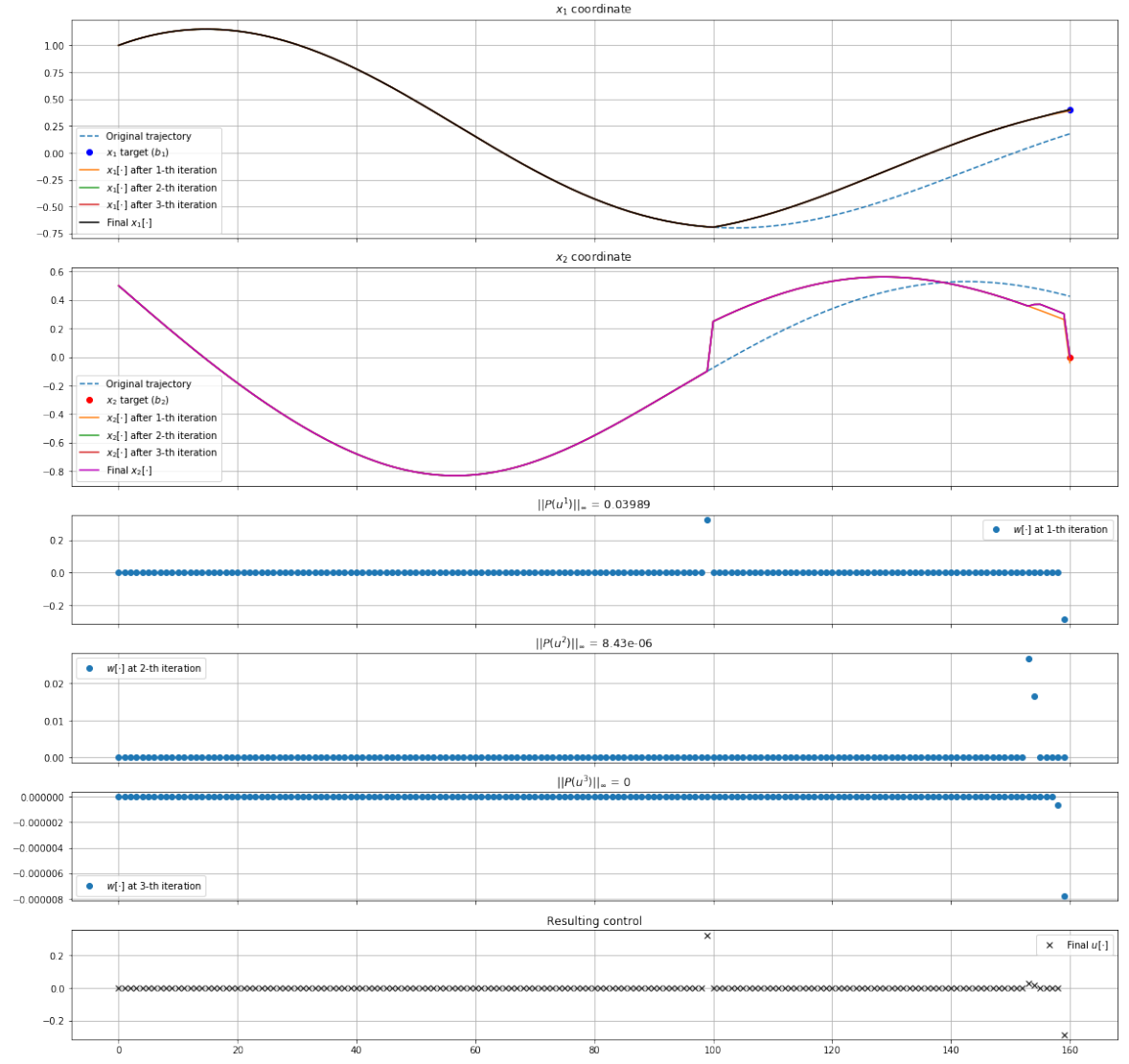

Fig. 1 Iterations of sparse Newton method 13: 5 non-zero components

\section{Conclusions}

We have proposed the novel approach for finding sparse solutions of boundaryvalue nonlinear dynamic problems, based on Newton-like method for solving under-determined systems of nonlinear equations. Practical simulation demonstrates high efficiency of the algorithm.

\section{Acknowledgements}

This work was supported by Russian Science Foundation, Project 16-11-10015. The authors thank the anonymous reviewers for their helpful comments.

\section{References}

1. Athans M.: Minimum-fuel feedback control systems: second-order case. IEEE Trans. Appl. Ind. 82, 8-17 (1963) 2 

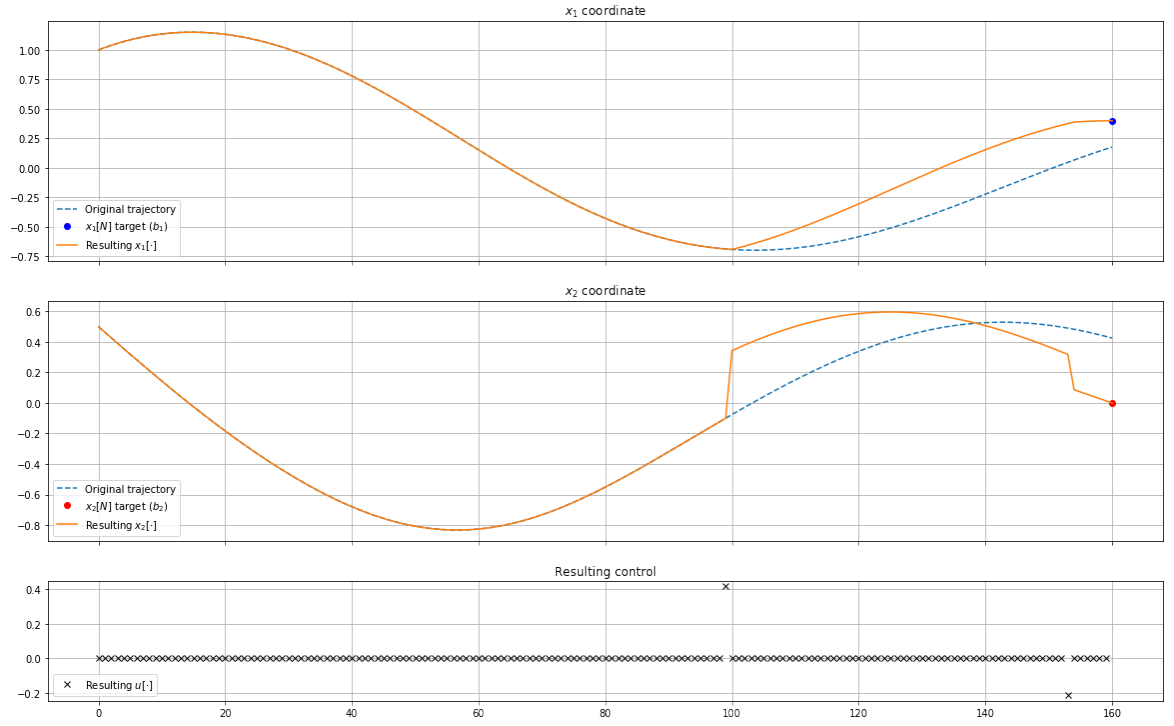

Fig. 2 Solution with two non-zero coordinates

2. Balakrishnan A.V., Neustadt L.W. (eds): Computing Methods in Optimization Problems. Academic Press, NY (1964) 1

3. Bellman, R.E.: Dynamic Programming. Princeton University Press, Princeton, NJ (1957) 1

4. Ben-Israel, A.: A Newton-Raphson method for the solution of systems of equations. J. Math. Anal. Appl. 15, 243-252 (1966) 5

5. Burdakov, O.P.: Some globally convergent modifications of Newton's method for solving systems of nonlinear equations. Soviet Math. Dokl. 22(2), 376-379 (1980) 4

6. Candes, E.J., Wakin, M.B., Boyd, S.P.: Enhancing sparsity by reweighted $\ell_{1}$ minimization. J. Fourier Anal. Appl. 14(5-6), 877-905 (2008) 7

7. Dennis, J.E., Schnabel, R.B.: Numerical Methods for Unconstrained Optimization and Nonlinear Equations. SIAM, Philadelphia (1996) 3

8. Evtushenko Yu.G.: Numerical Optimization Techniques. Springer, NY (1985) 1

9. Hager, W.W.: Analysis and Implementation of a Dual Algorithm for Constrained Optimization. J. Optim. Theory Appl. 79(3), 427-462 (1993) 5

10. Kantorovich, L.V., Akilov, G.P.: Functional Analysis. 2nd ed. Pergamon Press, Oxford (1982) 4,6

11. Kelley, C.T.: Solving Nonlinear Equations with Newton's Method. SIAM, Philadelphia (2003) 3

12. Lee E.B., Markus L.: Foundations of Optimal Control Theory. John Wiley, NY (1967) 1

13. Leitman G. (ed.): Optimization Techniques. Academic Press, NY (1962) 1

14. Leondes C.T. (ed.): Control and Dynamic Systems. Advances in Theory and Application, volume 16. Elsevier (1980) 1

15. Levin, Y., Ben-Israel, A.: A Newton method for systems of $m$ equations in $n$ variables. Nonlinear Anal. 47, 1961-1971 (2001) 5

16. Nesterov, Yu.: Modified Gauss-Newton scheme with worst case guarantees for global performance. Optim. Methods Softw. 22(3), 469-483 (2007) 5

17. Nagahara M., Quevedo D.E., Nešić D.: Maximum hands-off control: A paradigm of control effort minimization. IEEE Trans. Autom. Control. 61(3), 735-747 (2016) 2

18. Ortega, J.M., Rheinboldt, W.C.: Iterative Solution of Nonlinear Equations in Several Variables. SIAM, Philadelphia (2000) 3,4 
19. Pan, B., Ma, Ya., Yan, R.: Newton-type methods in computational guidance. J. Guidance, Control Dyn. 42(2), 377-383 (2018) 2

20. Polak E.: Computational Methods in Optimization: A Unified Approach. Academic Press, NY-London (1971) 1

21. Polyak, B.T.: Gradient methods for solving equations and inequalities. USSR Comput. Math. Math. Phys. 4(6), 17-32 (1964) 4

22. Polyak, B.T.: Newton-Kantorovich method and its global convergence. J. Math. Sci. 133(4), 1513-1523 (2006) 4

23. Polyak, B., Tremba A.: Solving underdetermined nonlinear equations by Newton-like method. Submitted to Optim. Methods Softw. (2018). https://arxiv.org/abs/1703.07810 2, 5, 6

24. Polyak, B., Tremba A.: Newton method with adaptive step-size for under-determined systems of equations. Proceedings of the VIII International Conference on Optimization and Applications (OPTIMA-2017), Petrovac, Montenegro, October 2-7, 475-480 (2017) 25

25. Rao, C.V.: Sparsity of linear discrete-time optimal control problems with $l_{1}$ objectives. IEEE Trans. Autom. Control. 63(2), 513-517 (2018) 2

26. Tabak, D., Kuo B.C.: Optimal Control by Mathematical Programming. Prentice-Hall (1971) 2

27. Walker, H.F.: Newton-like methods for underdetermined systems. In: Allgower, E.L., Georg K. (eds.) Computational Solution of Nonlinear Systems of Equations, Lecture Notes in Applied Mathematics. 26, 679-699, AMS, Providence, RI (1990) 5

28. Yamamoto, T.: Historical developments in convergence analysis for Newton's and Newton-like methods. J. Comput. Appl. Math. 124, 1-23 (2000) 4 